\title{
Preventing COVID-19 Infection in a University Using Office 365
}

\author{
Shigekazu Katagiri \\ katagiri@fukuyama-u.ac.jp \\ Fukuyama University \\ Fukuyama, Japan \\ Kazuki Yoshizu \\ Fukuyama University \\ Fukuyama, Japan
}

\author{
Takashi Yamanoue \\ yamanoue@fukuyama-u.ac.jp \\ Fukuyama University \\ Fukuyama, Japan \\ Shinji Hira \\ hira@fukuyama-u.ac.jp \\ Fukuyama University \\ Fukuyama, Japan
}

\begin{abstract}
Preventing COVID-19 infection in a university using a cloud computing service, Microsoft Office 365, is discussed. In order to start in-person classes safely, our university decided to survey health status of every student and staff member, every day. The student or the staff member is asked to not to come to campus if there is a symptom of COVID-19 infection or if they did not fill out the survey sheet daily for two weeks. If there are no symptom of COVID-19 for two weeks, there is almost no possibility of infection of COVID-19 or the person had recovered from the infection. The survey data is collected and analyzed with Office 365 to minimize time and effort. In addition to the survey, we made the class seat assignment sheets using our academic affair system and Excel. The assigned seats were used in order to coduct contuct tracing if a student tested positive with COVID-19.
\end{abstract}

\section{CCS CONCEPTS}

- Social and professional topics; • Applied computing $\rightarrow$ Enterprise applications;

\section{KEYWORDS}

COVID-19, infection prevention, robotic process automation, cloud computing

ACM Reference Format:

Shigekazu Katagiri, Takashi Yamanoue, Kazuki Yoshizu, and Shinji Hira 2021. Preventing COVID-19 Infection in a University Using Office 365. In ACM SIGUCCS Annual Conference (SIGUCCS '21), March 14-April 30, 2021, Virtual Event, USA. ACM, New York, NY, USA, 6 pages. https://doi.org/10. $1145 / 3419944.3441219$

\section{INTRODUCTION}

The prevalence of COVID-19 affects activities of universities in world wide in 2020. Many campuses were closed and online classes

Permission to make digital or hard copies of all or part of this work for personal or classroom use is granted without fee provided that copies are not made or distributed for profit or commercial advantage and that copies bear this notice and the full citation on the first page. Copyrights for components of this work owned by others than ACM must be honored. Abstracting with credit is permitted. To copy otherwise, or republish, to post on servers or to redistribute to lists, requires prior specific permission and/or a fee. Request permissions from permissions@acm.org.

SIGUCCS '21, March 14-April 30, 2021, Virtual Event, USA

(c) 2021 Association for Computing Machinery.

ACM ISBN 978-1-4503-8141-3/21/03 . \$ \$15.00

https://doi.org/10.1145/3419944.3441219 were held instead of in-person classes during spring of 2020 [8]. Some universities started in-person classes after that.

Our university decided to postpone the start of classes until May 2020, after the new fiscal year in April. Online classes were started in May.

The crisis response headquarters of our university announced the health survey, on April 8th, 2020, in order to have in-person classes safely, once the danger for infection was mitigated.

Every student and staff member are asked to fill out their health status, every day in the survey. The student or the staff member is asked to not come to campus if they have symptoms of a COVID-19 infection or if they did not fill out the survey sheet daily for two weeks. If there are no symptoms of COVID-19 for two weeks, there is almost no possibility of infection of COVID-19 or if the person had recovered from the infection. In the survey, the body temperature, other health statss, places visited, the place of residence, and any comment for concern were surveyed.

The survey was implemented utilizing Microsoft Office 365 in order to collect and analyze the data, with the goal of minimizing time and effort.

We also made the class seat assignment sheets using our academic affairs system and Excel. This was used to narrow the range of the infection of COVID-19. If a student tested positive with COVID19 , we could do contact tracing. We made a small VBA macro for automating the generation of class seat assignment sheets.

We could know the potential of COVID-19 infection and we could cope with the situation earlier using the survey. When a student tests positive with COVID-19, we could narrow the range of other potentially infected people in our campus immediately using the survey and the seat assignment sheets.

\section{HEALTH SURVEY SYSTEM}

\subsection{Outline}

Figure 1 shows the flow of the survey system. Students and staff member fill out their health status in the survey utilizing Office 365 Forms.

The source sheet, which contains the list of all of the health statuses, are generated automatically by Office 365 . The source Excel sheet is converted into a temperature table sheet, the comment list, and the submission status list sheet. Some of them are converted automatically using Excel functions. The temperature table sheet contains the table which shows the body temperature of every person of every day. When the value of the temperature exceeds 


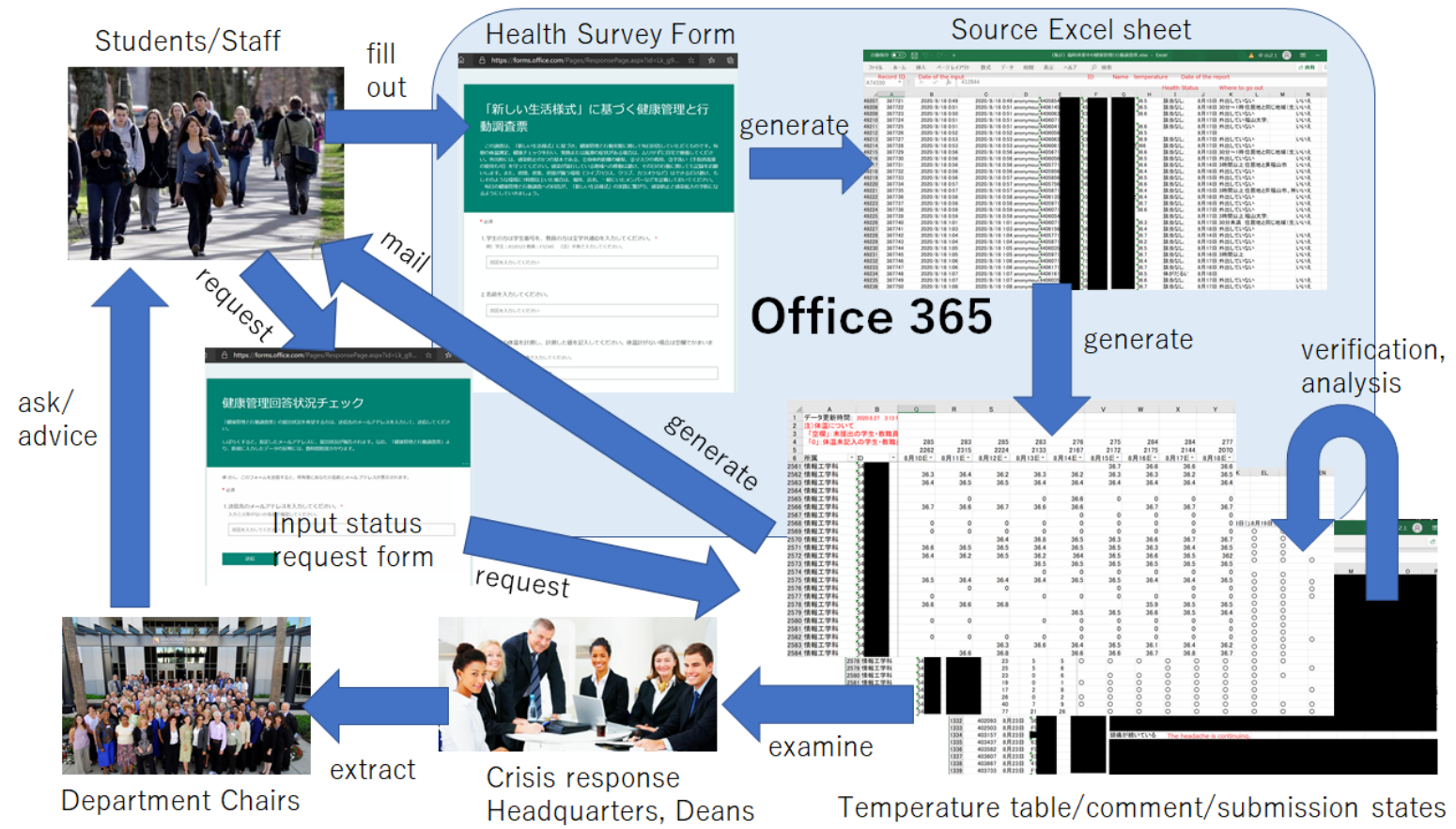

Figure 1: Flow of the survey.

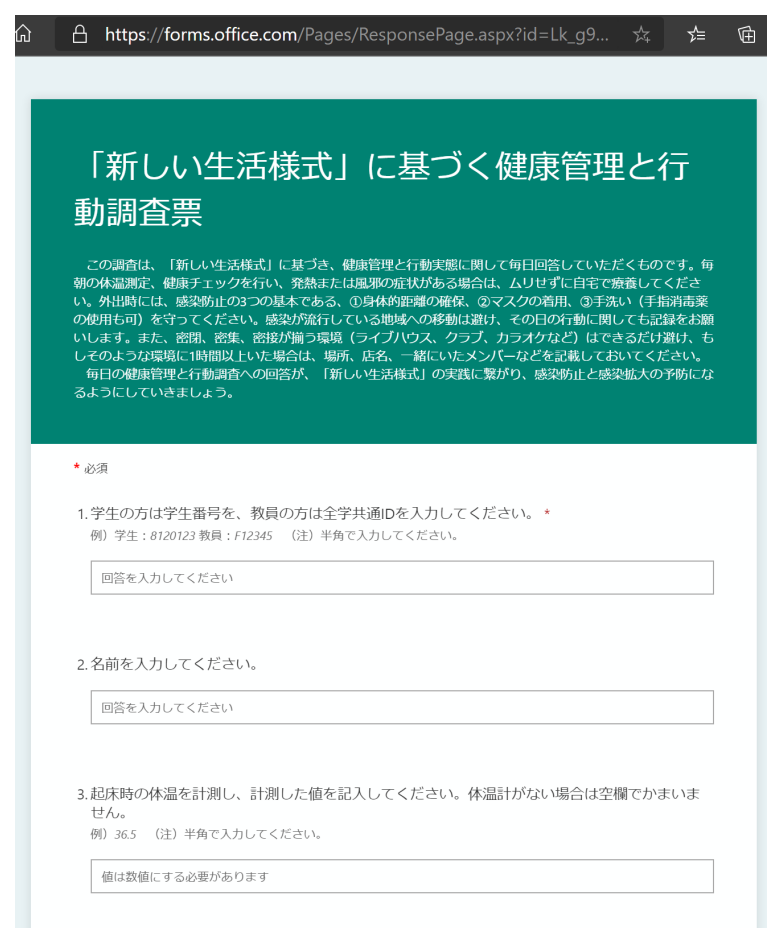

Figure 2: Survey form.

37.5 degree Celsius, or 99.5 degrees Fahrenheit, the value is marked for review automatically.
The comment list sheet contains the list of comments. This list is generated by hands. The submission status list sheet contains the list of who fills out the health status when.

There may be mistakes in values. So anomalies are corrected every day by the person who is in charge of the survey.

These sheets are examined by the crisis response headquaters and passed to deans of schools of our university every day. Every dean extracts their departments' data for their school from the sheets and they pass the data to their departments' chairs.

When the crisis response headquarters notes something wrong in the sheets, the headquarters request the department chair to investigate the health status of the student or staff reporting the symptoms.

When a department chair finds an anomaly in the data which was not found by the headquarters, the chair asks the person whose health status shows possible infection.

Students or staff members may forget, or they can not to fill out the survey every day. Everyone is allowed to fill out the survey of a day after they realized that they forgot to fill out the survey the day before. In order to fill out the survey of the previous day, the person requests their survey records. The person's records are generated from the submission status list sheet, and the list is sent to the person by e-mail.

\subsection{Survey form}

Figure 2 shows the survey form. This survey form is a Web page and this page is linked to our university's web site. 


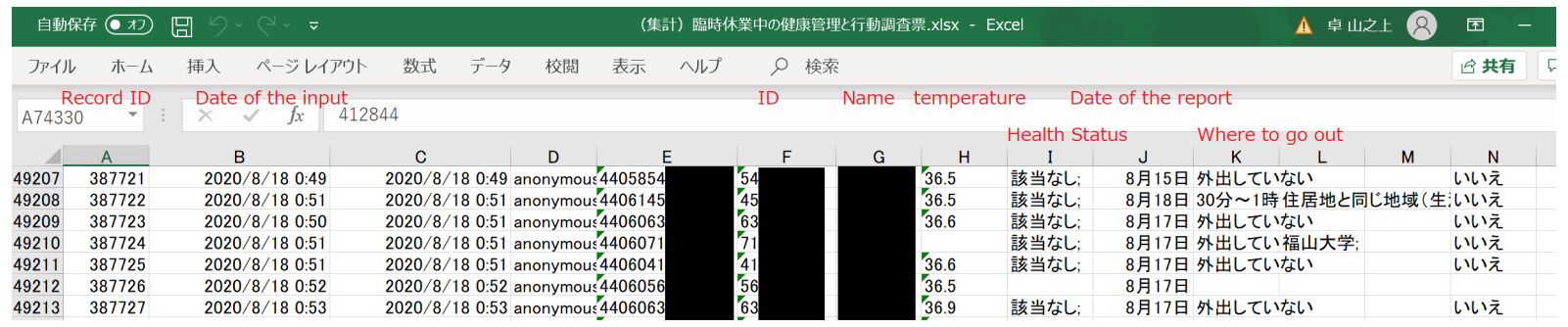

Figure 3: Source sheet.

\subsection{Source sheet}

Figure 3 shows a part of the source Excel sheet. This sheet is generated automatically by Office 365 . Column A of the sheet shows the submission number individual filling out survey. Column B and C show the date and the time the survey was completed. Column $\mathrm{E}$ and $\mathrm{F}$ show the ID number of the student, faculty or staff member who filled out the survey. Column G shows the name of the person. Column $\mathrm{H}$ shows the body temperature at the time the survey was completed. Column I shows the health status of the person, other than their body temperature. Column J shows the date of the health status of the person. Column K shows whether the person goes out or not. Column $\mathrm{N}$ shows whether the person's current residence is on the University residence list or not.

\section{$2.4 \quad$ Temperature table sheet}

Temperature table sheet is generated from the source sheet using Excel functions. Each row of the sheet shows a person. Column A of the sheet shows the department of the person who filled out the survey. Column B shows the ID number of the person. Column C shows the name of the person. The range from column $\mathrm{R}$ onward shows the body temperature of the person of day. The date is each entry in row 6 . For example, the person in row 2556 their body temperature was 36.2 Celsius(97.2 Fahrenheit) on August 12th. The blank cell in this range shows that the person did not fill out the survey form on that date of the listed column. The "0" cell shows that the person filled out the survey form on that date of the column; however, the person could not measure their temperature on that specific day. The members of the crisis response headquarters read temperatures in this table every day and direct the deans and the department chairs, who are in charge of the person, to cope with the situation when the temperature listed is higher than 37.5 Celsius.

\subsection{Comment List sheet}

Column A of the comment list sheet shows the submission number of the entry. Column B shows the date the survey was completed. Column $\mathrm{C}$ shows the ID number of the individual who filled out the survey. Column D shows the name of the person. Column E shows any comments. In this figure, the comment of row 1239 shows that the person went to a hospital and had a PCR test. The members of the crisis response headquarters read these comments and direct the deans and the department chairs, who are in charge of the person, to address the situation when required.

\subsection{Submission status list sheet}

The submission status list seet is generated from the source sheet using Excel functions. Each row of the sheet shows a person. Column A of the sheet shows the department of the person who filled out the survey. Column B shows the ID number of the person. Column $\mathrm{C}$ shows the name of the person. The range from column ED and the followings columns show if the person filled out the survey on a particular day which is shown by each column in the seventh row. The blank cell in this range shows that the person did not filled out the survey form on the particular date at the top column. The circle in the cell in this range shows that the person filled out the survey form on the date of the column. For example, the person of the row 2556 did not filled out the survey on August 11th and filled it out on August 12th.

This sheet was prepared for deans and department chairs in order for them to know how many students and faculty members of their school or departments filled out the survey, without leaking private information such as the body temperature and thier comments. After the survey started, there were many requests from students and faculty for the means to know when they filled out the survey form them-selves.

In order to respond to this request, the crisis response headquarters had to add the submission status list request and response system to the survey system.

After that, this sheet was also used when someone would like to know when a person filled out the survey form and requested the list of the dates completed. The list of values on the line which is corresponding to the person is returned to the requester after that. This request and response system was made using the "power automate" [4] function of Office 365. Figure 5 shows an example of the returned e-mail after the request made by the filling out the form. Figure 4 shows the form for requesting the submission status list for a person. The required field for filling out is the person's e-mail address only. The person's ID number is assigned when the person $\log$ into the Office 365 in order to open this form.

\section{CLASS ROOM SEAT ASSIGNMENT}

We would like to reduce the chance of infection with COVID-19 while we have in-person classes. Even if a student has tested positive with COVID-19, we would like to track the past places where the student has visited and we would like to narrow the range of people who could also possibly to be infected with COVID-19 utilizing contact tracing. 


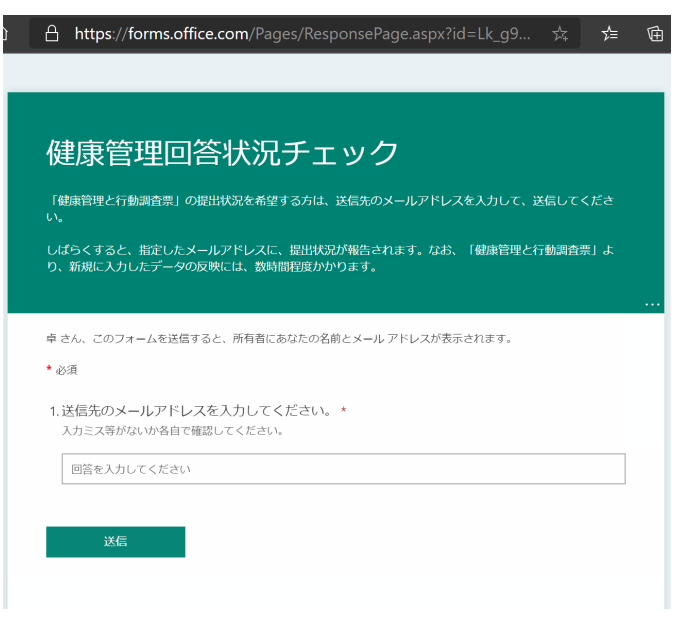

Figure 4: Submission status list request form.

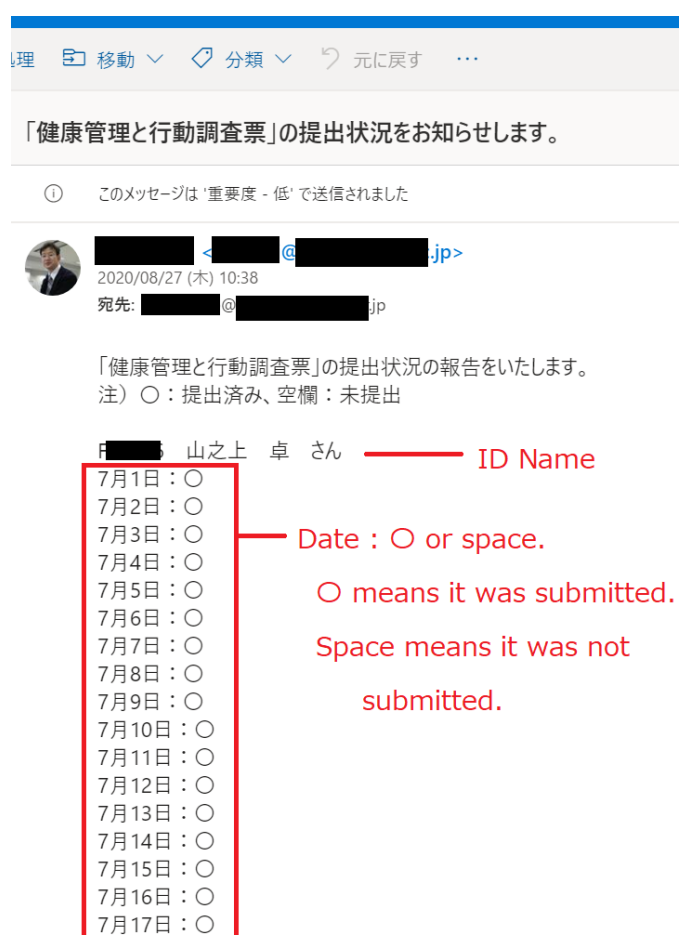

Figure 5: Example of the returned e-mail after the request.

In order to minimize contact, we made the class room seat assignment sheets, in addition to the health survey system. Every student has to take their assigned seat for every in-person class at our university when we started the in-person classes on June 22th. This was after the national and local government restrictions to prevent the spread of infection were lifted.

There were almost no assigned seats before the prevalence of COVID-19. After that, in order to reduce the chance of infection of COVID-19, we assigned seats for every student in every class and made a space between nearby seats. Figure 6 shows the picture of a class where every student took their assigned seat.

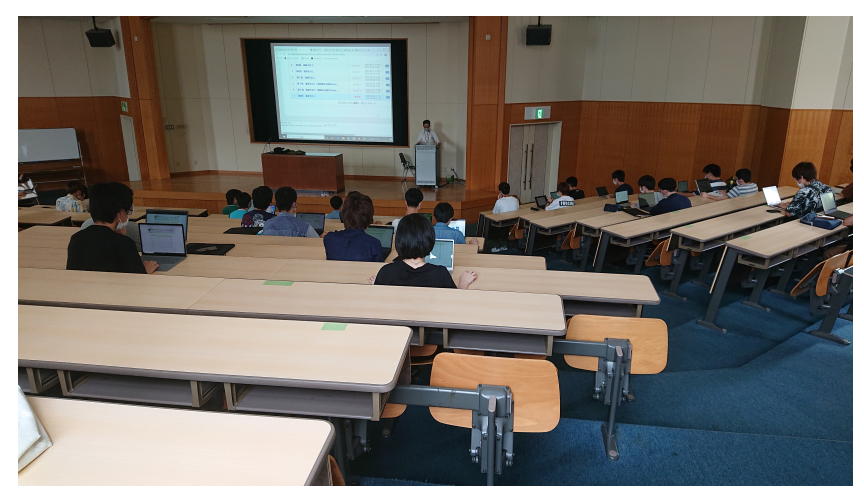

Figure 6: Seat assignment in a class

In order to have a space between nearby seats, it was impossible to have all in-person class. Some large classes were continued to be online classes. Even if the number of in-person classes was reduced, there were about five hundred in-person classes. That meant, that, we had to make five hundreds class room seat assignment sheets.

It is almost impossible to make all the sheets by hands in a limited time frame. We had automate the process to make the sheets using the class seat assignment sheets generator.

The generator inputs the Excel file, which contains the class registration information for every student, and Microsoft Word files, which contain the seat map for every class room. This outputs the class room seat assignment sheet for every class for every student.

The Excel file was downloaded from our academic affairs system.

As the first stage of making the file generator, we had make the generator using e-mail merge function of Microsoft Word 365. The generator at this stage generates a class room seat assignment sheet for a class in a Microsoft Word file, by connecting the Excel file as the data base for e-mail merge, and by choosing the class room name, the week of the class, and the time of the class, by hand. The Microsoft Word file contains the seat map of the class room.

The e-mail merge function reduced the time for the generating seat assignments. However, it was very cumbersome to choose each class room by hand.

At the second stage of making the seat assignment generator, we made a VBA macro which mimics manual input form the first stage of thegenerator. Figure 7 shows the flow of the classroom seat assignment sheets generator. Figure 8 shows an example of the generated seat assignment sheet.

\section{OTHER MEASURES}

In addition to the aforementioned measures, we did the following:

- We placed sanitiz alcohol dispensers at every entrance of every building and asked everyone, who enters the building, to sanitize their hands.

- We placed some packages of sanitizing wipes in every computer laboratory. Students were asked to clean the keyboard and mouse with the sanitizing wipes before each use. There 


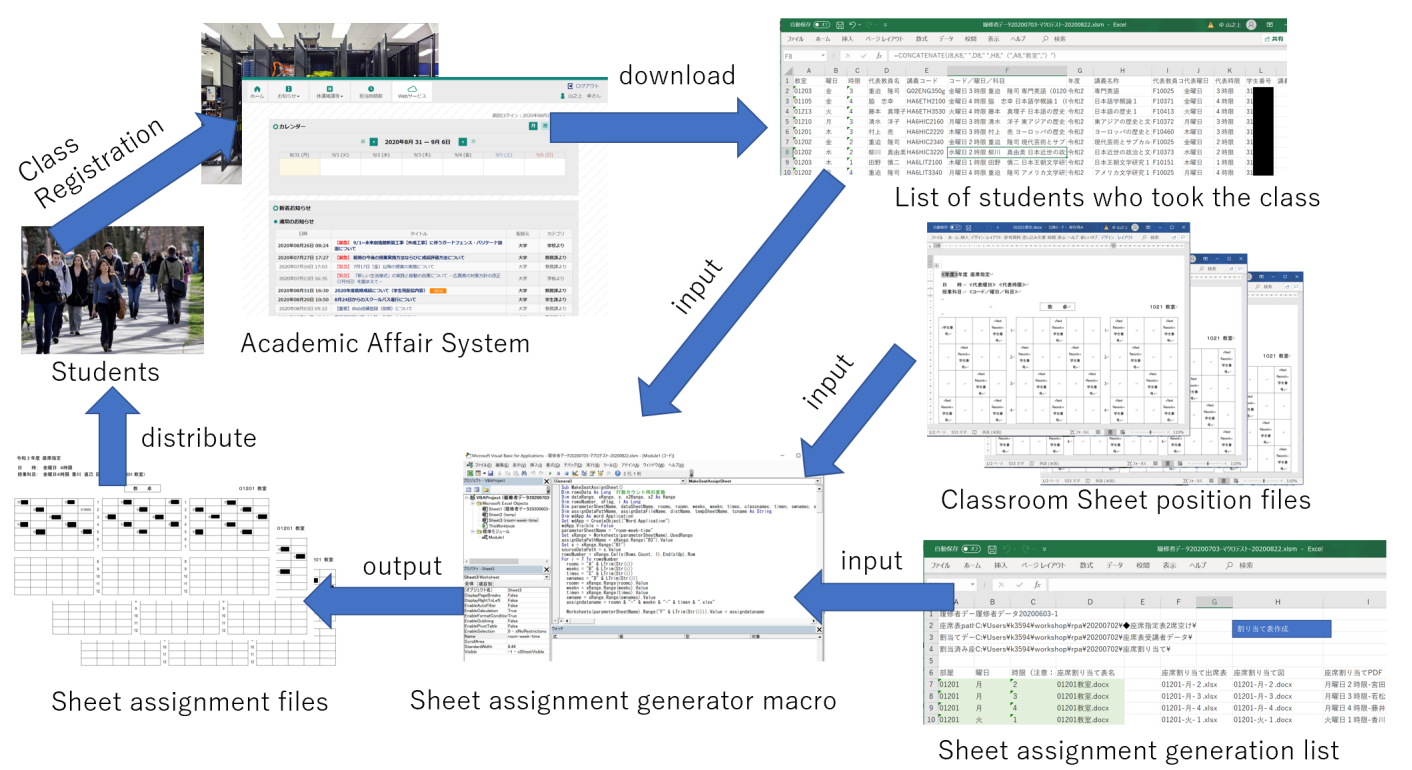

Figure 7: Flow of the classroom seat assignment sheets generator

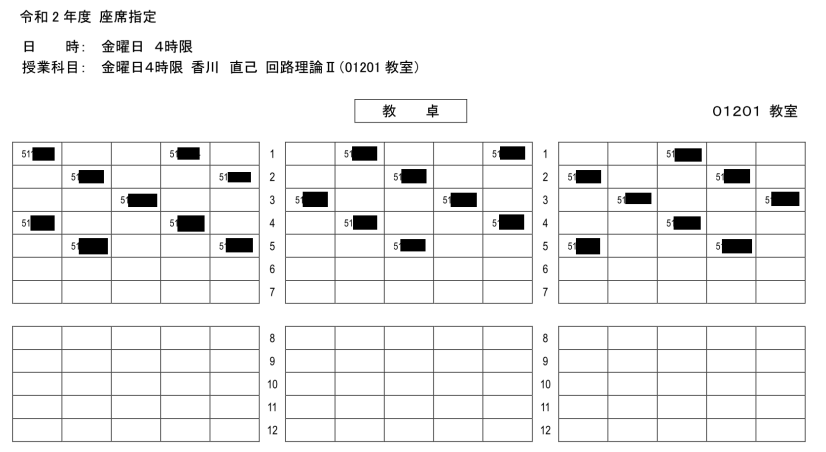

Figure 8: Seat assignment in a class

have been cases of COVID-19 infection due to touching common items such as door knobs, computer touchpads and other objects.

- We asked faculty to open classroom windows frequently in order to avoid the classroom being sealed. Airflow has proved important to mitigate COVID-19 infections.

- Many of our students commute to schooling using our school buses. In order to avoid crowding on a bus, the bus schedule was re-scheduled and the beginning of the first class of the day was delayed. Every class time was reduced from 90 minutes to 80 minutes.

\section{RESULTS}

There was no infections until July 16th 2020 at our university. One student was found to be infected with COVID-19 at that time. He was following common guidelines to mitigate COVID-19 infection, and the student's contacts were traced. Unfortunately, he did not keep filling out the survey form every day. When he was found to be infected by COVID-19, we could narrow the range of other potentialy infected people in our campus immediately using the survey and the seat assignment sheets. After that, there were no infections of COVID-19 in our university until October 2020.

Our university have been recognized by Fukuyama city as an institution which has complied with the guidelines of the Fukuyama city's measures against the COVID-19 infection September 4th 2020.

\section{RELATED WORK}

\subsection{COCOA, COVID-19 Contact Confirming Application}

The Japanese ministry of health, labor and welfare released its coronavirus contact tracing app for iOS and Android, COCOA, June 19th [1]. The apps rely on Apple and Google's co-developed exposure notification platform, using Bluetooth to help determine whether users have come into close contact with others who have tested positive for COVID-19.

There was a case that COCOA notified a user who came into close contact with a COVID-19 infected person, and the user was also found infected with COVID-19. They were tested due to the notification. Any new infection of COVID-19 could be prevented if notified people do not go out or stayed in a hospital for two weeks [2]. COCOA is useful in preventing further COVID-19 infections.

We ask that all students, faculty and staff members install COCOA on their smart phones. 


\subsection{COVID-19 Testing in UIUC}

Faculty, staff and students at the University of Illinois UrbanaChampaign who will regularly be on campus this fall must get tested for COVID-19 twice a week [6].

This testing must be very effective to prevent COVID-19 infection. We would like to consider adopting such testing methods as they might prove useful in our situation.

\subsection{The Science Council of Japan Suggestions}

The science council of Japan suggests the promotion of strengthening Information and Communication Technology (ICT) infrastructure and digital transformation for infectious disease countermeasures and society transformation [5]. The suggestion includes (1) the digital transformation of medical systems, (2) the digital transformation of living society, and (3) privacy protection and cyber security.

Our ICT countermeasures are also using ICT infrastructure and digital transformation. We would like to show that ICT infrastructure and digital transformation are effective for preventing disease infection, and we believe it has shown to be effective by our experiences until now.

\subsection{Robotic Process Automation}

Robotic Process Automation (RPA) [7] is an application of technology, governed by business logic and structured inputs, aimed at automating business processes. There are many RPA tools in the world now and they are very similar to Excel macro.

Our university has automated a part of the assessment of our university using a kind of RPA [3].

The countermeasures against COVID-19 infection in this paper use the "Power Automate" [4] function of Office 365. The Power Automate is a tool of RPA.

\section{CONCLUDING REMARKS}

We have shown our countermeasures against COVID-19 using a cloud computing service, Microsoft Office 365 . It has shown effective so far. However, there are many things that could improve our ICT countermeasures. For example, there are still many things that need to be done by hand, and our system works in batches, not real time. We would like to improve such problems, especially, because it is annoying for users to fill out the survey form every single day. We are considering automating the survey.

\section{ACKNOWLEDGMENTS}

We would like to thank all of our faculty staff and students who are cooperating to fill out our survey and for their continued patience.

\section{REFERENCES}

[1] Labor Japanese Ministry of Health and Welfare. 2020. COCOA, Covid-19 COntact Confirming Application. https://www.mhlw.go.jp/stf/seisakunitsuite/bunya/ cocoa_00138.html.

[2] Yasushi Ohkusa Junko Kurita, Tamie Sugawara. 2020. Effectiveness of COCOA, a COVID-19 contact notification application, in fapan. medRixiv. https://doi.org/10. 1101/2020.07.11.20151597

[3] Yoshitaka Kihira, Takashi Yamanoue, Motoo Tanaka, Eiji Sato, and Yutaka Otsuka. 2019. Automating a Part of the Assessment of a University Using Excel. In Proceedings of the 2019 ACM SIGUCCS Annual Conference (New Orleans, LA, USA) (SIGUCCS '19). Association for Computing Machinery, New York, NY, USA, 76-81. https://doi.org/10.1145/3347709.3347821

[4] Critchley S. 2020. Power Automate. Apress, Berkeley, CA. https://doi.org/"10. 1007/978-1-4842-5911-5 13"

[5] The Science Council of Japan 2020. The Suggestion of the Promotion of Strengthing ICT Infrastructure and Digital Transformation for Infectious Disease Coutermeasures and Society Transformation. The Science Council of Japan. http://www.scj.go.jp/ ja/info/kohyo/kohyo-24-t298-3-abstract.html.

[6] University of Illinois Urbana-Champaign 2020. ON-CAMPUS COVID-19 TESTING. University of Illinois Urbana-Champaign. https://covid19.illinois.edu/health-andsupport/on-campus-covid-19-testing/.

[7] Martin Bichler Willibrordus Martinus Pancratius van der Aalst and Armin Heinzl. 2018. Robotic Process Automation. https://doi.org/"10.1007/s12599-018-0542-4

[8] Alexandra Witze. 2020. Universities will never be the same after the coronavirus crisis. nature. https://doi.org/10.1038/d41586-020-01518-y 